\title{
Interview With Yu (Lucy) Huang, Coordinator, Data Strategy \& Fan Loyalty, National Basketball Association China
}

\author{
Wei-Yen Li \\ Indiana University-Bloomington, USA
}

After pursuing an international education, Yu Huang, a native of China, returned to her homeland to commence her career in the sport industry. Huang, who is the coordinator of Data Strategy \& Fan loyalty with the National Basketball Association China (NBA China), is based in Shanghai, China. Before she joined NBA China, Huang was a specialist in sport-event management at YooZoo Sports, a company that uses internet space operation (ISO) to develop high-tech infrastructure in stadiums. In addition to YooZoo Sports, the YooZoo Group has several other subsidiaries (e.g., YooZoo Games, YooZoo Picture). Currently, YooZoo Sports has three large multipurpose sport parks that concentrate on soccer practices and games but also provide use for other sports (e.g., Shanghai YXSpace). Revenue is also generated by renting the facilities to private companies in Shanghai. Huang's role with YooZoo Sports involved developing marketing and partnership opportunities with corporations and leagues around the world. Some examples of these partners include Fan Zhiyi, a former professional soccer player who played for the Chinese national team and professional teams (e.g., Shanghai Greenland Shenhua Football Club) and the Great Stone Gridiron Corporation (GSG). GSG is a company based in China that aims to introduce American football to China and develop interest in the sport through hosting youth camps and initiatives, partnering with elementary schools and building training facilities in Beijing and Shanghai. After her time with YooZoo Sports, Huang joined NBA China and assumed her current coordinator role, working with the data-strategy and fan-loyalty program NBA Qiu Mi Quan (QMQ), the first NBA fan-loyalty program. Her primary responsibilities are maintaining QMQ platforms, launching regular online campaigns, and organizing fan events.

Li: As a coordinator of the data-strategy and fan-loyalty program with NBA China, what is the most interesting and challenging parts of this position?

Huang: The new program itself. My colleagues and I here at NBA China can try different campaigns to interact with fans. Trying various approaches makes my

The author is with the Dept. of Kinesiology, Indiana University, Bloomington, IN. Address author correspondence to li591@iu.edu. 


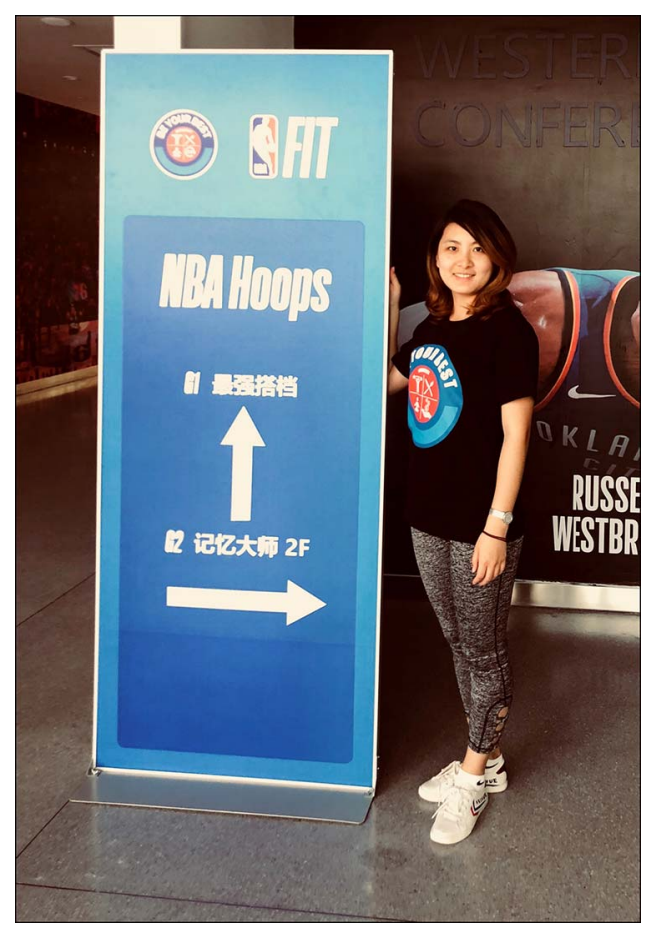

Yu (Lucy) Huang) - Coordinator, Data Strategy \& Fan Loyalty, National Basketball Association China.

position and work here interesting. Some examples of our campaigns include making dumplings with Jeremy Lin and fans' playoff bracket predictions, which provide an opportunity for Chinese participants to win a playoff ticket. The interesting part of these campaigns is a dream come true for Chinese NBA fans and myself because we get the opportunity to interact with NBA players in person. However, my work as a coordinator can sometimes be challenging because my colleagues and I at times encounter numerous obstacles and failures before we finally discover how the program works. For example, we found that during some of our campaigns we have had a difficult time increasing fan participation rates to levels that we had expected in light of the NBA's high brand awareness in China.

Li: What is NBA Qiu Mi Quan (QMQ)? How does this program build fan loyalty, and can you provide an example or two of how the program works?

Huang: NBA QMQ is the first NBA fan-loyalty program and is specifically focused on the Chinese market. In addition, the NBA introduced the program in China as a method for market testing. If the program achieves huge success in China, the NBA will implement the program in other countries. As for how the program works, NBA QMQ has a rewards system that encourages fans to watch, 
play, and compete on any Internet-accessible platform such as a computer or a mobile device. To be more specific, fans can register an account on the NBA QMQ website (https://www.nbaqmq.com/) and earn points by interacting with other registrants from China and finishing daily missions (e.g., daily login; watching NBA games through Tencent Sports, an online live distributor; correctly predicting the scores of NBA games). Then, fans can use their reward points for online badges that will show up on their profiles. The reward points can be used to purchase NBA merchandise (e.g., jerseys, hats) and secure coupons for the NBA Store.

Li: As a relatively new project for the NBA, are there any unique obstacles faced by NBA QMQ, and what would you consider some of the early successes of this program?

Huang: NBA QMQ faces challenges because my colleagues in NBA China and I need to try out the market and see how fans react to our campaigns regarding this fan-loyalty program. The major TV network in China, CCTV5, and online live distributors (e.g., Tencent Sports) have helped us accumulate a lot of registered fans, and the various fan bases of the teams (e.g., Los Angeles Lakers, Cleveland Cavaliers) and stars (e.g., Kobe Bryant, LeBron James) have participated in the program, as well. Thus, the NBA does not have any problems attracting Chinese fans. However, the way for the NBA to engage with fans is a pending test. It is a good thing that the NBA is aware of the importance of fan loyalty and has local agencies working together. For example, KLUE is our content-creation agency, which is made up of local sport lovers who know how to attract Chinese fans and help NBA China to attract thousands of new registrations daily. On the successful side, we have nearly 1.5 million registered fans for the NBA QMQ program. We also have good partnerships with global businesses to run the fan campaigns, which generate media attention. For example, NBA China launched a campaign with Chivas, which is called “CHIVAS·NBA 就是燃," an event promotion in which Chivas looked to infuse the NBA's exciting, competitive, and emotional image into Chivas' original brand image to attract a younger segment. The event associated with CHIVAS-NBA was hosted in Shanghai and included Paul Pierce, a former NBA star player, and Shawn Yue, a Chinese celebrity. The campaign not only helped Chivas increase its whiskey sales and brand awareness in China, but the campaign also benefited NBA China as it provided market buzz and a multiyear marketing partnership with Chivas.

\section{Li: What do you envision for the future for NBA QMQ?}

Huang: To become the biggest and most influential fan-loyalty program in the global sport industry. This is a lofty goal, but it is a realistic goal, and thus I can envision this happening. There are two reasons for my optimism. First, few-if any-professional leagues around the world are as aware of, interested in, or invested in fan data as the NBA. Second, the NBA has many initiatives in the global market (e.g., it recently expanded its effort into South Africa) and is dedicated to building worldwide fame. I believe that with the NBA's effort in the global market it can easily implement NBA QMQ in other countries because it has already built a foundation. 
Li: As professional leagues around the world are trying to enter the market in China, what are your thoughts about their attempts and the opportunities and challenges therein? Related to this, how is NBA China preparing to deal with this?

Huang: The National Football League (NFL) and the National Hockey League (NHL) have tried for many years and in different ways to tap into the Chinese market, but their respective fan bases have not been sufficient to make an immediate impact in the market. It takes years, decades, and even generations to grow a significant fan population. Also, American football and ice hockey are still new to the Chinese people, meaning there are both low participation and viewing rates for these sports in China. In comparison, basketball is the largest sport in China, with great fans, as the NBA has now been in China for 20 years. Meanwhile, NBA legends such as Michael Jordan and Kobe Bryant have had a great impact in China. What's more, former NBA great Yao Ming is an icon in China, which leads a lot of youngsters to become loyal fans of the NBA. Thus, the NBA has had an easier time generating people's interest and is considered one of the most influential sport leagues in China. However, the NBA still has a long way to go when it comes to localizing. What I mean by localizing is to increase fan engagement and brand awareness in China. Regarding the localization effort of the league, the NBA partners with 3,000 Chinese high schools in the Junior NBA program, which brings an advanced basketball training program to the young people attending Chinese schools.

Li: Does your department (Data Strategy \& Fan Loyalty) and/or NBA China work independently from the NBA (e.g., conceive ideas and projects and then report to the NBA) or does the NBA instruct certain or all goals, projects, and plans to be executed by NBA China and your department?

Huang: Employees in NBA China can work independently but need to get approval from the NBA and are required to report to the league, as NBA China is financially supported by the NBA. For instance, we need to get approval from the NBA for our campaigns and report to the NBA regarding our various initiatives. In addition, NBA QMQ is under the strategy department of NBA China.

Li: Yao Ming is chairperson of the Chinese Basketball Association League Sports Company (CBA company), which hosts the highest level of professional league (i.e. CBA league), and the president of the Chinese Basketball Association (CBA), which focuses on basketball development in China. With his international stature, what do you see as the major impact of his involvement with the CBA company and CBA league, and will that involvement affect the NBA in its international ambitions?

Huang: Yao Ming and the NBA have a good relationship. With his successful career with the Houston Rockets, the NBA gained a considerable number of Chinese fans and built a solid foundation for the league's expansion in China. In his role as chairperson of CBA company, Ming focuses more on that particular league, but I am sure he and the NBA have more to work on together in China. In addition to Ming's focus on CBA, he currently intends to change game rules and involve more sponsorships around the world. 
Li: Many NBA teams have established an eSport team by playing NBA $2 \mathrm{~K}$. At the beginning of 2017, the CEO of NBA China, David Shoemaker, expressed an interest in having an NBA eSport league, which may be separate from the established NBA or incorporated into it. What are your thoughts on an eSport league and the NBA's involvement?

Huang: The NBA has an NBA 2K league that launched this year (2018). NBA China and Tencent have launched an eSport basketball game called 最强 (the best) NBA, which is popular among Chinese sport gamers and hosts competition nationally, and have put much effort into this eSport league. For example, in 2018, 最强 NBA hosted its national college competitions under University Cyber League, the biggest eSport competition among Chinese college students. During the finals, former NBA player Ray Allen was invited to the stage and introduced a new version of the game; as a result, the NBA attracted 6 million people to watch the finals.

\section{Li: How is YooZoo Sports involved with the ISO?}

Huang: ISO is the core value of YooZoo Sports. I stands for Internet. YooZoo Sports inherits the Internet genre of YooZoo Games in tech development and bigdata analysis. $S$ stands for space. YooZoo Sports is building its own national sports complex. $O$ stands for operation. Operation is the core of making a complex be sustainable to the company and the public. YooZoo hires operating experts as the directors.

Li: When you worked with YooZoo Sports, your company negotiated a partnership with the Pacific-12 (Pac-12) Conference, one of the more popular Division I intercollegiate athletic conferences affiliated with the National Collegiate Athletic Association (NCAA) in the United States. What can you tell me about that negotiation and your experiences as a part of that initiative?

Huang: YooZoo Sports developed the business with the Pac-12 and discussed the potential events (e.g., tennis invitational games, Chinese sport carnivals) for almost a year. However, YooZoo Sports did not find a way to implement the events. There are two reasons for the implementation challenges. First, Alibaba is the Pac-12's main sponsor and partner; thus, Alibaba has the main right Pac-12 China's games (i.e., Pac-12 has annually invited two or three universities to compete in seasonal men's basketball games in China since 2015). YooZoo Sports may develop new event projects that Alibaba does not want to be involved with, but new projects need time and money to be carried out, which is a huge risk for any business. Second, the Pac-12 has a limited budget for global development. In the end, all current events are supported by Alibaba.

Li: Could you talk about the experiences you had as you developed business opportunities with the local sport industry as a Great Stone Gridiron (GSG) corporation?

Huang: GSG was a big success during my time with YooZoo Sports. The corporation signed a 3-year contract with the YooZoo West Bund soccer park. 
At the very beginning, the complex was conceived as a facility only for soccer. However, my YooZoo Sports colleagues and I found out that the a new complex's utilization rate was low during the weekdays. Thus, I figured that involving sports other than soccer and creating long-term partners would be the solution to the utilization-rate problem. As a business developer, I began to investigate the various options and to examine various sport organizations in China in my attempt to seek potential cooperation opportunities in order to achieve my plan. Because of my previous experiences with both NFL China and the Indianapolis Colts of the NFL, I was able to secure a good relationship with the GSG manager. After I introduced the ideas to him, he had the same eagerness to find a longtime partner. Then, we set up the first GSG-West Bund campus in Shanghai.

Li: YooZoo is one of the largest game-development and -distribution companies in China. However, there is not much of a YooZoo footprint in eSports. Could you share your thoughts on why this is the case and what you see as YouZoo's role and plans for eSports involvement in China and beyond?

Huang: The main domain of YooZoo Games involves games offered through the Internet and personal computers. The company also created mobile games but not eSports. I think the reason for not venturing into eSports is that eSport gaming needs more time and money to develop and maintain. The business of eSports is not one in which money is quickly generated. Return on investment (ROI) for eSports is not as lucrative as PC/Web gaming for the company. On the other side, YooZoo is now working on buying a Chinese eSports team and building an eSports training base in Shanghai.

Li: Several universities in the United States give athletic scholarships to eSports athletes. Do you see this type of activity ever happening in China or do you see eSports as strictly recreational or professional in nature, with no direct connection to higher education? Also, what are your thoughts regarding eSports overall in China?

Huang: Some of the universities in China also have an eSport department. Furthermore, the government in China encourages the professionalization of eSports. The General Administration of Sport of China also assigns the informatics center to regulate eSports and to build up a national team. China already has a prosperous eSport league, called the King Pro League (KPL), which is hosted by Tencent, one of the largest IT companies in China. With 3.6 billion views of KPL during the season, I think that the KPL is promising and will be expanded globally. However, it depends on whether the game itself goes viral. In addition, China has the Chinese E-sports Football League and a developing league, the eSport Basketball Association. I do see an increased eSports presence in the Chinese sport industry. For example, I can see more and more professional teams coming out and new technology such as virtual reality and augmented reality being significantly integrated into the eSports experience and expansion.

Li: Why did you join NBA China as the next step in your career? 
Huang: Besides Nike and Adidas, I can say that the NBA is the most successful global sport brand in China. The NBA has a mature business model with localization experience about which I would like to learn. NBA China also follows the creative trend in initiatives (e.g., the NBA is trying to combine basketball with eSport, as well as involving virtual reality/augmented reality into NBA games), which I find interesting, and I could see myself working in those areas in the future.

Li: Could you give some advice to people who are looking to enter the sport industry? In addition, would you mind sharing advice regarding those who might want to work in the sport industry in China and maybe even with NBA China?

Huang: My advice is to try different things such as being involved with different positions and sports, to think actively, and to stay patient. The sport industry in China is not as mature as it is in the United States. People need to find out what will work in China, and during the process, they are going to experience thousands of challenges. It is hard to taste the fruit of success instantly, but you are always growing with the industry. It is not a place to earn quick money, but you should always hang in there. Develop, practice, and become proficient with your skills (e.g., basic computer programs, verbal and nonverbal communication, basic data analysis) and build up your contacts and networks, and you will eventually find something that works out. 